\title{
Características clínicas, uso de recursos y costos directos de pacientes con EPOC de una institución de salud de Bogotá
}

Yudy Medina ${ }^{1}$, Jaime Alvarado ${ }^{2}$, Juan Iriarte ${ }^{2}$, Adriana Castañeda ${ }^{2}$, Yully Guerrero ${ }^{3}$, Carmen Garcia ${ }^{4}$, Maria José Fernández ${ }^{4}$, Andrés González ${ }^{4}$ (1) Instituto para la Evaluación de la Calidad y Atención en Salud IECAS, (2) CLIINICOS Programas de atención integral SAS IPS, (3) Universidad de La Sabana, (4) GlaxoSmithKline Colombia

Introducción

En Colombia la EPOC es la tercera causa de muerte en mayores de 45 años y la segunda causa de consultas al sistema de salud entre las enfermedades crónicas de las vías respiratorias inferiores (1).

Objetivo

Describir las características clínicas, uso de recursos y costos directos de la atención de la EPOC en una institución prestadora de salud de Bogotá.

Métodos

- Diseño: Estudio descriptivo con recolección retrospectiva de datos.

- Población: Se incluyeron pacientes del régimen subsidiado del programa integral de EPOC de una IPS entre 2015 y 2016 con espirometría confirmatoria e información clínica disponible.

- Información clínica: Las características clínicas basales, evolución clínica y uso de recursos de los pacientes en un periodo de 12 meses fueron extraídos de las historias clínicas de la institución. Se aplicó la clasificación GOLD 2011 a partir de la función pulmonar y el MMRC basales.

- Costos: Para el cálculo del costo directo se multiplicó la frecuencia medida de uso de recursos por los precios publicados en manuales tarifarios SOAT, ISS con ajuste de $30 \%$ y la base de datos SISMED.
Resultados

$\checkmark$ Se incluyeron 535 pacientes, 84\% mayores de 65 años (tabla 1).

$\checkmark$ No se observó una tendencia entre la categoría GOLD y el uso de recursos en esta muestra, excepto por una mayor frecuencia de exacerbaciones en los grupos B y D (tabla 2).

$\checkmark$ La media geométrica del costo directo anual de la atención de la EPOC para las categorías GOLD A, B, C y D fue respectivamente $\$ 892.761$ (IC95\% 491.212 a 1.294.311), \$1.327.458 (IC95\% 1.088.414 a 1.566.503), $\$ 1.234 .184$ (IC95\% 322.083 a 2.146.285) y $\$ 2.838 .112$ (IC95\% 2.302.653 a 3.373.571).

$\checkmark$ Un $88 \%$ del costo total correspondió a manejo de exacerbaciones (tabla 3).

Conclusiones

El tratamiento de las exacerbaciones fue el principal determinante del

costo directo de la EPOC. El costo total del grupo GOLD D es

significativamente más alto que los demás

Punto de discusión

El dato de exacerbaciones previas no estaba disponible; algunos pacientes con clasificación GOLD B por función pulmonar y sintomas podian ser GOLD D.

\begin{tabular}{|c|c|c|c|c|c|c|c|c|c|c|c|c|c|c|c|c|}
\hline \multicolumn{6}{|c|}{ Tabla 1: Caracteristicas demográficas y clinicas } & \multicolumn{6}{|c|}{$\begin{array}{l}\text { Tabla 2: Desenlaces clinicos y uso de recursos en salud según grupo } \\
\text { GOLD basal }\end{array}$} & \multicolumn{5}{|c|}{$\begin{array}{l}\text { Tabla 3: Promedio anual de los costos directos } \\
\text { de la EPOC según categoria y grupo GOLD }\end{array}$} \\
\hline Clasfificación GOLD 2011 & $\begin{array}{r}\text { GOLDA } \\
(n=52)\end{array}$ & $\begin{array}{l}\text { GOLD B } \\
(n=273)\end{array}$ & $\begin{array}{l}\text { GOLDC } \\
(n=14)\end{array}$ & $\begin{array}{l}\text { GOLD D } \\
(n=196)\end{array}$ & $\begin{array}{r}\text { Total } \\
(n=535)\end{array}$ & Clesificacion GOLP & & & & & & \multirow{2}{*}{$\begin{array}{l}\text { Grupo GOLD } \\
2011\end{array}$} & \multirow{2}{*}{$\begin{array}{r}\text { GOLDA } \\
(n=52)\end{array}$} & \multirow{2}{*}{$\begin{array}{r}\text { GOL B } \\
(n=273)\end{array}$} & \multirow{2}{*}{$\begin{array}{r}\text { GOLDC } \\
(n=14)\end{array}$} & \multirow{2}{*}{$\begin{array}{l}\text { GOLD D } \\
(n=196)\end{array}$} \\
\hline Edad & $72(7,5)$ & $77(12)$ & $61,5(16)$ & $71(13)$ & $74(13)$ & 2011 & $(n=5$ & $(n=2$ & $(n=1$ & $(n=1$ & $\begin{array}{r}100 a 1 \\
(n=535)\end{array}$ & & & & & \\
\hline Sexo femenino & $37(71,1)$ & $168(61,6)$ & $8(57,1)$ & $96(49)$ & $309(57,8)$ & Visitas & & & & & & \multirow{4}{*}{$\begin{array}{l}\text { Visitas al } \\
\text { médico } \\
\text { especialista" }\end{array}$} & & & & \\
\hline Indice de masa corporal" & $26(7,9)$ & $26(7,3)$ & $22(4,2)$ & $23(7,5)$ & $25(7,8)$ & & $2(0-8)$ & $3(0-12)$ & $4(1-9)$ & $3(0-11)$ & $3(0-12)$ & & 51.266 & 71.275 & 4.235 & \\
\hline Tiempo en el & \multirow{3}{*}{$35(21,5)$} & \multirow{3}{*}{$35(25)$} & \multirow{3}{*}{$31(19)$} & & & Card & $0(0,0)$ & & & & & & & 43.969 & 37.617 & 42.885 \\
\hline $\begin{array}{l}\text { (meses) } \\
\text { (n) }\end{array}$ & & & & \multirow{2}{*}{$38(22,5)$} & \multirow{3}{*}{$36(23)$} & Gerontologia & $4(7,7)$ & $19(7,0)$ & $0(0,0)$ & $12(6,1)$ & $35(6,5)$ & & & & & \\
\hline Estrato socloeconómlco & & & & & & Medicina interna & $37(71,1)$ & $192(70,3)$ & $8(57,1)$ & $135(68,9)$ & $372(69,5)$ & \multirow{2}{*}{$\begin{array}{l}\text { Exámenes } \\
\text { diagnósticos }\end{array}$} & 24.953 & 31.470 & 44.481 & 33.473 \\
\hline Estrato 1 & $5(9,6)$ & $39(14,3)$ & $0(0,0)$ & $30(15,3)$ & & Neumología & $31(59,6)$ & $166(60,8)$ & $14(100,0)$ & $142(72,4)$ & $353(66,0)$ & & 18.660 & 26.000 & 37.267 & 27.099 \\
\hline Estrato 2 & $3(5,8)$ & $11(4,0)$ & $0(0,0)$ & $10(5,1)$ & $24(4,5)$ & $\begin{array}{l}\text { Toxicologia } \\
\text { Endocrinolog }\end{array}$ & $1(1,9)$ & $9(3,3)$ & $1(7,1)$ & $10(5,1)$ & $21(3,4)$ & \multirow{2}{*}{$\begin{array}{l}\text { Tratamiento } \\
\text { farmacológico }\end{array}$} & 240.784 & 324.645 & 494.866 & 537.005 \\
\hline Estrato 3 & $0(0,0)$ & $2(0,8)$ & $0(0,0)$ & $1(0,5)$ & $3(0,6)$ & nica & $(5,8)$ & $24(8,8)$ & $\begin{array}{l}0(0,0) \\
0\end{array}$ & 16 & $\begin{aligned} 01 \\
43(8\end{aligned}$ & & 183.961 & 254.777 & 392.246 & 431.187 \\
\hline Sin infor & $44(84,6)$ & $221(80,9)$ & $14(100,0)$ & $155(79,1)$ & $434(81,1)$ & Medicina fisica y del & & & & & & \multirow[b]{2}{*}{ Exacerbaciones } & 2007250 & 2831333 & 2216500 & 4792821 \\
\hline Función pulmonar basal & & & & & & deporte & $0(0,0)$ & $2(0,7)$ & $0(0,0)$ & $0(0,0)$ & $2(0,4)$ & & 3.757 .572 & $\begin{array}{l}2.0303 .350 \\
4.397 .197\end{array}$ & 4.140 .662 & 5.411.629 \\
\hline VEFY $\geq 8$ & $5(9,6)$ & $19(7,0)$ & $0(0,0)$ & $0(0,0)$ & $24(4,5)$ & Vistias otros & & & & & & & & 3.258 .722 & $2,820,080$ & $5.437,600$ \\
\hline $50 \% \leq V$ & $47(90,4)$ & $254(93,0)$ & $0(0,0)$ & $0(0,0)$ & $301(56,3)$ & professlo & $0(0-1)$ & $0(0-3)$ & $0(0-0)$ & $0(0-2)$ & $0(0-3)$ & Total & $\begin{array}{r}2,324,253 \\
892,761\end{array}$ & $\begin{array}{l}3,200,722 \\
1,327,458\end{array}$ & $1,234,184$ & 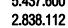 \\
\hline $30 \% \leq$ VEF 1 & $0(0,0)$ & $0(0,0)$ & $13(92,9)$ & $166(84,7)$ & $179(33,5)$ & Nutrición & $\begin{array}{l}0(0,0) \\
2(38)\end{array}$ & $\begin{array}{r}1(0,3) \\
19(7,0)\end{array}$ & $\begin{array}{l}0(0,0) \\
0(0,0)\end{array}$ & $\begin{array}{r}1(0,5) \\
10(5,1)\end{array}$ & $\begin{array}{r}2(0,4) \\
31(5,8)\end{array}$ & & & & & \\
\hline VEF $1<30 \%$ & $0(0,0)$ & $0(0,0)$ & $1(7,1)$ & $30(15,3)$ & $31(5,8)$ & Psicologia & $2(3,8)$ & $19(7,0)$ & $0(0,0)$ & & & & & & & \\
\hline $\begin{array}{l}\text { Caminata de } 6 \text { minutos } \\
\text { (m) }\end{array}$ & $372(145)$ & $344(152)$ & 495 (114) & 322 (159) & 343 (153) & $\begin{array}{l}\text { Pruebas diagnósticas" } \\
\text { Espirometria }\end{array}$ & $\begin{array}{r}3(0-16) \\
0(0,0)\end{array}$ & $\begin{array}{r}4(0-15) \\
7(2,6)\end{array}$ & $\begin{array}{r}4(2-13) \\
0(0,0)\end{array}$ & $\begin{array}{r}4(0-17) \\
7(3,6)\end{array}$ & $\begin{array}{l}4(0-17) \\
14(2,6)\end{array}$ & $\begin{array}{l}\text { Para el cálculo de } \\
\text { pacientes con co }\end{array}$ & osto cero. & métricas se & xcluyeron & \\
\hline $\begin{array}{l}\begin{array}{l}\text { Resultados CAT basal" } \\
\text { Impacto leve }(<10)\end{array}\end{array}$ & & & & & & Oxigeno & $4(7,7)$ & $11(4,0)$ & $3(21.4)$ & $18(9,2)$ & $36(6,7)$ & $\begin{array}{l}\text { pacientes con cos } \\
\text { *Incluye costo } \mathrm{d}\end{array}$ & de rehabilitació & ión pulmonar & & \\
\hline $\begin{array}{l}\text { Impacto leve }(<10) \\
\text { Impacto medio }(10-20)\end{array}$ & $\begin{array}{r}27(87,1) \\
4(129)\end{array}$ & $\begin{array}{r}87(42,6) \\
105(51,5)\end{array}$ & $\begin{array}{l}5(71,4) \\
2(28,6)\end{array}$ & $\begin{array}{l}44(28,2) \\
93(59,6)\end{array}$ & $\begin{array}{l}163(40,9) \\
204(51,3)\end{array}$ & & & & & & & & & & & \\
\hline Impacto al & $\begin{aligned} 4(1,9,9) \\
0(0,0)\end{aligned}$ & $11(5,4)$ & $\begin{array}{r}(20,0) \\
0(0,0)\end{array}$ & $16(10,3)$ & $\begin{array}{r}27(6,8) \\
27(1,3)\end{array}$ & ICS/LABA & $3(5,8)$ & $26(9,5)$ & $4(28,6)$ & $41(20,9)$ & $74(1$ & & & & & \\
\hline $\mathrm{Imp}$ & $0(0,0)$ & $1(0,5)$ & $0(0,0)$ & $3(1,9)$ & 411 & LAMALL & $0(0,0)$ & & $0(0,0)$ & & & & & & & \\
\hline & & & & & & (1) & $7(13,5)$ & $84(30,8)$ & $9(64,3)$ & $108(55,1)$ & $208(38,9)$ & & & de exacerb & thaciones ye & versus \\
\hline basal' & & & & & & LABA & $0(0,0)$ & $0(0,0)$ & $0(0,0)$ & $1(0,5)$ & $1(0,2)$ & &  & sto anual & baciones ve & versus \\
\hline MMRC 0 & 5,8) & $0(0,0)$ & $3(21,4)$ & $0(0,0)$ & $6(1,1)$ & SAMA & $51(98,1)$ & $264(96,7)$ & $14(100,0)$ & $189(96,4)$ & $518(96,9)$ & & & & & \\
\hline MMRC 1 & $49(94,2)$ & $0(0,0)$ & $11(78,6)$ & $0(0,0)$ & $60(11,3)$ & 10 & $\begin{array}{l}36(69,2) \\
32(615)\end{array}$ & $195(71,4)$ & $13(92,9)$ & $173(88,3)$ & $417(77,9)$ & 35 & & & & . \\
\hline MMRC 2 & $0(0,0)$ & $175(64,6)$ & $0(0,0)$ & $86(43,9)$ & $261(49$ & OCS & $\begin{array}{l}\begin{array}{r}2(61,5) \\
3(5,8)\end{array} \\
\end{array}$ & $\begin{array}{r}203(4,4,4) \\
18(6,6)\end{array}$ & $\begin{array}{r}11(78,6) \\
1(7,1)\end{array}$ & $\begin{array}{r}165(84,2) \\
12(6,1)\end{array}$ & $\begin{array}{r}411(16,8) \\
34(6,4)\end{array}$ & 30 & & & & $t$ \\
\hline MMRC 3 & $0(0,0)$ & $90(33,2)$ & $0(0,0)$ & $83(42,3)$ & $173(32,5)$ & Frecuenci & & & & & & & & & & $:$ \\
\hline MMRC 4 & $0(0,0)$ & $6(2,2)$ & $0(0,0)$ & $27(13,8)$ & $33(6,2)$ & exacerba & 3) & & 5) & $1(0-11)$ & $1(0-$ & & & & $:$ & \\
\hline Comorbllildades & & & & & & 0 & & & & & 220 & & & & . & \\
\hline ICC & $6(11,5)$ & $49(17,9)$ & $0(0,0)$ & $27(13,8)$ & $82(15,3)$ & 1 & $17(32,7)$ & & $4(28,6)$ & $56(28,6)$ & 157 & & & & & \\
\hline Neuropatia diabética & $2(3,8)$ & $33(12,1)$ & $1(7,1)$ & $16(8,2)$ & $52(9,7)$ & 2 & $4(7,7)$ & $36(13,2)$ & $1(7,1)$ & $36(18,4)$ & 771 & 15 & & & & \\
\hline Enfermedad coronaria & $6(11,5)$ & $22(8,1)$ & $0(0,0)$ & $11(5,6)$ & $39(7,3)$ & $\begin{array}{l}3 \\
40 \text { más }\end{array}$ & $\begin{array}{l}4(7,7) \\
0(0,0)\end{array}$ & $17(6,2)$ & $0(0,0)$ & $21(10,7)$ & & & & . & & \\
\hline Fibrilación auricular & $1(1,9)$ & $11(4,0)$ & $0(0,0)$ & $11(5,6)$ & $23(4,3)$ & & & $13(4,8)$ & $1(7,1)$ & $25(12,8)$ & $39(7,3)$ & & 1 & & & \\
\hline Asma & $1(1,9)$ & $7(2,6)$ & $0(0,0)$ & $3(1,5)$ & $11(2,1)$ & . & s. & . & . & uscarinio & 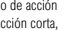 & 1 & 1 & & & \\
\hline $\begin{array}{l}\text { VEF1: volumen espiratorio } \\
\text { MMRC Modififed Medical } \\
\text { Las variables están expresa } \\
\text { intercuartil) } \\
*^{*} n=532 \text { para indice de mas } \\
\text { CAT } n=535 \text { para MMRC. }\end{array}$ & $\begin{array}{l}\text { ado en el } \\
\text { arch Cou }\end{array}$ & $\begin{array}{l}\text { primer se } \\
\text { ncili, ICC: } \\
\text { cuencia } p\end{array}$ &  & $\begin{array}{l}\text { PDD asses } \\
\text { rdiaca cor } \\
\text { diana (rat }\end{array}$ & & $\begin{array}{l}\text { * Mediana por persona (rar } \\
\text { † Visitas a urgencias u hos } \\
\text { pacientes inicialmente clasis } \\
\text { más exacerbaciones al año } \\
\text { muestran según su clasific }\end{array}$ & $\begin{array}{l}\text { 1). Las demás v } \\
\text { lizaciones por } \\
\text { ados como GO } \\
\text { eron reclasificar }\end{array}$ & $\begin{array}{l}\text { ariables están e } \\
\text { EPOC ocuridas } \\
\text { LD Ay B por Su } \\
\text { dos como GOLL }\end{array}$ & 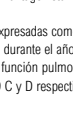 & $\begin{array}{l}\text { de conieo } \\
\text { de obser } \\
\text { nar que } \\
\text { vamente }\end{array}$ & unos & 01 & ${ }_{\text {Nim }}^{2}$ & $\begin{array}{ll}4 & 5 \\
\text { de exacertac: } & 6 \\
\end{array}$ & 789 & 9 \\
\hline
\end{tabular}

Declaraciones: Este estudio fue patrocinado por GSK, código H0-15-16339. CG, MJF y AG son empleados y/o accionistas de GSK. YM recibió honorarios por el proyecto de investigación, pero su actuar científico fue independiente al patrocinio. AC, YG, JA y JI no tienen conflictos de interés.

\section{Referencias}

1. Ministerio de Salud y Protección Social, Organización Panamericana de la Salud. Morbi-mortalidad de las enfermedades respiratorias crónicas, Colombia 2005-2013 2016. 